\title{
Impact of an acute coronary syndrome (ACS) specialist nurse service to reduce time to coronary angiography +/-
} revascularisation

\author{
Authors: Tijo Mathew, Michelle Hayes, Hannah Fenn, Hadleigh Cutbert, Clare Carter-Jones, Roby Rakhit \\ and Tim Lockie
}

\begin{abstract}
Aims
To assess whether the introduction of an acute coronary syndrome (ACS) clinical nurse specialist (CNS) service reduces the time to coronary angiogram +/- revascularisation in non-ST-elevation myocardial infarction (NSTEMI) patients in the setting of an acute urban teaching hospital.
\end{abstract}

\section{Methods}

We each collected 6 months' data for NSTEMI patients admitted before (2016) and after (2017) the introduction of the ACSCNS service. The audit analysed the time from admission to angiography for both groups. A system called infoflex is used in the hospital to record the details about angiograms and percutaneous coronary interventions. The data about NSTEMI was collected electronically from this system. Then, the patients who met the exclusion criteria were excluded. The exclusion criteria include type 2 NSTEMI (ischaemic myocardial necrosis due to supply-demand mismatch, eg coronary spasm, low blood pressure, anaemia, or arrhythmias) and patients unfit for angiography for other medical comorbid reasons including pulmonary oedema or sepsis. 112 and 100 patients were eligible for the study in 2016 and 2017, respectively.

\section{Results}

The audit showed that the median time to angiogram was $2 \pm 2.1$ days in 2016 in comparison to $1 \pm 1.2$ days in 2017 since the implementation of the ACS-CNS service. Furthermore, $59 \%$ of NSTEMI patients had angiogram within 72 hours in 2016 whereas, $92 \%$ of the patients had angiogram within 72 hours in 2017.

Patients presenting with NSTEMI are a significant burden on the acute medical take. Although current NICE guidelines advise that patients should have angiography within 72 hours of hospital admission, only half of all patients in the UK achieve this due to delays in diagnosis, risk assessment and access to the cath lab for angiography.

\section{Conclusion}

The audit showed that ACS-CNS service significantly reduced the time to angiography $+/$ - revascularisation. Also, it improved early evidence-based treatment for patients being admitted with ACS as per National Institute for Health and Care Excellence recommendation. Furthermore, it reduced the length of stay in hospital for ACS patients. As a result of this study, the trust has decided to continue ACS-CNS service. Moreover, this information has been presented in a few conferences to encourage other trusts to start ACS-CNS service.

\section{Conflict of interest statement}

Out of seven authors, two of them were acute coronary syndrome clinical nurse specialists. 